\title{
A CLASS OF FLEXIBLE NILSTABLE ALGEBRAS
}

\author{
WILLIAM G. WITTHOFT ${ }^{1}$
}

\section{Introduction. Algebras satisfying the identity}

$$
x(x a)+(a x) x=2(x a) x
$$

have been studied by Kosier [2] and by the author [3]. When such an algebra is strictly power-associative over a field of characteristic prime to six then, modulo its radical, it has an identity and is a direct sum of simple algebras. The simple algebras of degree no less than three are Jordan or quasi-associative. Those of degree two and nilstable were shown in [3] to be noncommutative Jordan algebras that are $J$-simple and consequently in possession of a multiplication table like that described by Kokoris [1]. In this paper we shall observe that when the ground field is ordered such an algebra is in fact Jordan and point out noncommutative examples of such algebras when the ground field is not ordered.

2. Let $A$, then, be a central simple strictly power-associative algebra of degree two, nilstable, and satisfying (1) over the field $F$. By [1], $A$ has a basis $B=\left\{e, f, a_{1}, a_{2}, \cdots, a_{n}\right\}$ for some $n \geqq 1$ where (i) $e$ and $f$ are orthogonal idempotents whose sum is the identity, 1 , of $A$, (ii) $e a_{i}=a_{i} / 2+\sum_{j} \alpha_{i j} a_{j}$ for each $i=1,2, \cdots, n$ where $\left\|\alpha_{i j}\right\|$ is a skew symmetric matrix over $F$, (iii) $a_{i} e=a_{i}-e a_{i}$ for each $i$, (iv) $a_{i}^{2}=1$ for each $i$, and $(\mathrm{v}) a_{i} a_{j}=2 \alpha_{i j}(e-f)+\sum_{k} \epsilon_{i j k} a_{k}$ for all distinct $i$ and $j$ where $\epsilon_{i j k}$ is skew symmetric in its indices and vanishes when two agree. Clearly then $f a_{i}=a_{i} e, a_{i} f=e a_{i}$, and $a_{j} a_{i}=-a_{i} a_{j}$ for all distinct $i$ and $j$. The new information here about multiplication in $A$ comes from substitutions of elements of $B$ into (1).

Theorem. If, for such an algebra $A, n \leqq 3$ or $F$ is ordered then $A$ is commutative.

First, (1) with $a$ replaced by $a_{i}$ and $x$ by $e$ produces $e\left(e a_{i}\right)+\left(a_{i} e\right) e$ $=2\left(e a_{i}\right) e$. Adding $e\left(a_{i} e\right)+\left(e a_{i}\right) e$ to both sides and using the facts that $A$ is flexible and $a_{i} e+e a_{i}=a_{i}$ yield $a_{i}=4\left(e a_{i}\right) e$, i.e.,

$$
a_{i}=a_{i}-4 \sum_{j, k} \alpha_{i j} \alpha_{j k} a_{k} .
$$

Thus, for each $i$ and $k, \sum_{j} \alpha_{i j} \alpha_{j k}=0$, i.e., $\left\|\alpha_{i j}\right\|^{2}=0$. In particular, the diagonal entries, $\sum_{j} \alpha_{i j} \alpha_{j i}=-\sum_{j} \alpha_{i j}^{2}$, of $\left\|\alpha_{i j}\right\|^{2}$ vanish. This is impossible for $n \leqq 3$ or $F$ ordered unless each $\alpha_{i j}=0$.

Received by the editors August 5, 1968.

${ }^{1}$ The author was supported by NSF grant GP-7130. 
Again, (1) with substitutions becomes

$$
a_{j}\left(a_{j} a_{i}\right)+\left(a_{i} a_{j}\right) a_{j}=2\left(a_{j} a_{i}\right) a_{j} .
$$

Now, for $i \neq j$ ( $A$ is clearly commutative when $n=1$ ), $a_{j} a_{i}=-a_{i} a_{j}$ is a linear combination of the remaining $a_{k}$ 's, and so all products in (2) are anticommutative. Thus

$$
\begin{gathered}
2\left(a_{i} a_{j}\right) a_{j}=-2\left(a_{i} a_{j}\right) a_{j}, \quad\left(a_{i} a_{j}\right) a_{j}=0, \\
\sum_{k, l} \epsilon_{i j k} \epsilon_{k j l} a_{l}=0,
\end{gathered}
$$

and so $\sum_{k} \epsilon_{i j k} \epsilon_{k j l}=0$ for all $i, j$, and $l$. In particular, $0=\sum_{k} \epsilon_{i j k} \epsilon_{k j i}$ $=-\sum_{k} \epsilon_{i j k}^{2}$, so that, when $F$ is ordered, each $\epsilon_{i j k}=0$. When $n \leqq 3$ then, by skew symmetry, each $\epsilon_{i j k}=0$ or $0=\sum_{k} \epsilon_{i j k}^{2}=\epsilon_{i j k}^{2}$ with $i, j, k$ distinct. The vanishing of all $\alpha_{i j}$ and $\epsilon_{i j k}$ is exactly the condition for commutativity in $A$.

The theorem fails when $n \geqq 4$ and $F$ is not ordered. For example, when char $F=p$ there exist $\alpha, \beta$, and $\gamma$ not all zero in $F$ for which $\alpha^{2}+\beta^{2}+\gamma^{2}=0$ ( $p$ is a sum of three squares unless $p \equiv 7 \bmod 8$, in which case $2 p$ is such a sum). It suffices to let

$$
\left\|\alpha_{i j}\right\|=\left\|\begin{array}{rrrr:c}
0 & \alpha & \beta & \gamma & \\
-\alpha & 0 & \gamma & -\beta & 0 \\
-\beta & -\gamma & 0 & \alpha & 0 \\
-\gamma & \beta & -\alpha & 0 & \\
\hdashline & 0 & & 0
\end{array}\right\|
$$

and $\epsilon_{i j k}=0$ for all $i, j$, and $k$ to produce a noncommutative algebra that satisfies (1). When char $F=0$ but $F$ is not ordered, -1 is a sum of squares. In case $-1=\lambda^{2}$ for some $\lambda \in F$ choose:

$$
\left\|\alpha_{i j}\right\|=\left\|\begin{array}{rrrr:c}
0 & 1 & \lambda & 0 & \\
-1 & 0 & 0 & \lambda & 0 \\
-\lambda & 0 & 0 & -1 & 0 \\
0 & -\lambda & 1 & 0 & \\
\hdashline & 0 & & 0
\end{array}\right\|
$$




\section{BIBLIOGRAPHY}

1. L. A. Kokoris, Flexible nilstable algebras, Proc. Amer. Math. Soc. 13 (1962), 335-340.

2. Frank Kosier, On a class of nonflexible algebras, Trans. Amer. Math. Soc. 102 (1962), 299-318.

3. W. G. Witthoft, $A$ class of nilstable algebras, Trans. Amer. Math. Soc 111 (1964), 413-422.

UNIVERSITY OF NEW HAMPSHIRE 